\title{
Concomitant Medication Use End Relative to Reference Period
}

National Cancer Institute

\section{Source}

National Cancer Institute. Concomitant Medication Use End Relative to Reference Period. NCI Thesaurus. Code C83227.

A characterization of the relationship of an concomitant medication use conclusion point in time to a reference point in time. 